\title{
Th2 cells and the IFN- $\gamma$ R1 subunit in early and advanced experimental periodontitis in rats; an immunohistochemical study
}

\author{
Janaína Cavalcante Lemos ${ }^{1}$, Bruno César de Vasconcelos Gurgel ${ }^{2}$, Roseana de Almeida Freitas ${ }^{3}$ \\ ${ }^{1}$ Universidade Potiguar - UnP, School of Dentistry, Department/Area of Periodontics, Natal, RN, Brazil \\ Universidade Federal do Rio Grande do Norte - UFRN, School of Dentistry, Department/Area of Periodontics, Natal, RN, Brazil \\ ${ }^{3}$ Universidade Federal do Rio Grande do Norte - UFRN, School of Dentistry, Department/Area: Oral Pathology, Natal, RN, Brazil
}

\begin{abstract}
Aim: To evaluate the involvement of Th2 cells in different periods of the active phase of experimental periodontal disease and expression of the R1 subunit of the receptor for IFN- $\gamma$ during the early and advanced progression of the disease. Methods: Experimental periodontitis was induced in 30 male Wistar rats by placing cotton ligatures around the mandibular first molars. The rats were then randomly assigned into two groups: $\mathrm{G} 1=15$ and $\mathrm{G} 2=15$, in group $\mathrm{G} 1$, ligatures were maintained for 2 days, a period that corresponds to the initial stage of periodontal disease in rats, in $\mathrm{G} 2$ ligatures were left for 15 days, a period that corresponds to the advanced stage of periodontal disease. The contra-lateral teeth served as controls (unligated). An immunohistochemical investigation of the gingival tissue was performed to detect the presence of the Th2 specific transcription factor (GATA3). Results: Light microscopy analysis revealed a decreased expression of GATA-3-positive cells when bone loss progressed. IFN- $\gamma$ R1 was detected at an early stage during the active phase of disease, but the expression of positive cells remained unaltered during the remaining period of the study. Conclusions: These results indicate that the Th2 response may have a protective role during the pathogenesis of experimental periodontal disease, and that the IFN- $\gamma$ R1 subunit may not be associated with periodontal disease progression.
\end{abstract}

Keywords: rats; periodontal diseases; Th1 cells; Th2 cells; cytokines; immunohistochemistry.

\section{Introduction}

Received for publication: January 21, 2015 Accepted: May 28, 2015

Correspondence to: Janaína Cavalcante Lemos Av. Amintas Barros 3735 Bl. C ap. 603 Bairro Lagoa Nova CEP: 59075-250 Natal RN Brasil Phone: +55 (084) 32015017 (084) 99855455 E-mail: nainalemos@gmail.com
Periodontal disease is the result of microbial aggression as well as metabolic and environmental factors and life-style habits influence the pathogenesis of this disease $^{1}$. Bacteria are capable of damaging directly the host tissue, but it can also be destroyed by inflammatory processes resulting from this aggression as consequence of the exaggerated immunoinflammatory and proteolytic processes ${ }^{2}$ that activate osteoclasts ${ }^{3}$. CD4 and CD8 T lymphocytes play an import role in the inflammatory response, as these cells may manage the profile of cytokines produced against an infectious agent ${ }^{4}$. Cytokines are mediators that, among other functions, can cause bone resorption and dissolution of the extracellular matrix. Progression of periodontal lesions is caused by dysregulation of molecules (cytokines) released by specific cell populations ${ }^{5}$.

CD4 T cells may assume different phenotypes under different stimulations, changing to Th1 and Th2 cells. They can be distinguished based on the profile of 
cytokines produced: Th1 cells produce characteristic cytokines such as IL-2, IFN- $\gamma$, TNF- $\beta$, IL-12, while active Th2 cells secrete IL-4, IL-5, IL-6, IL-10 and IL-13 ${ }^{6}$. In addition to Th1 and Th2 cells, Th17, Treg and T Fh cells have also been described ${ }^{7}$, Treg cells are associated with reduction of clinical scores of disease in soft and hard tissues ${ }^{8}$. Usually, protective and destructive roles are assigned to the Th1 and Th173,9-11 cells, while Th2 and Treg cells are more involved in processes that reduce the destruction of the periodontium ${ }^{10}$.

In order to differentiate into Th1 or Th2 cells, CD4 T cells must become antigen-activated. Differentiation into Th1 depends on the presence of IFN- $\gamma$ and IL-12, which bind to receptors on the surface of CD4 $\mathrm{T}$ cells ${ }^{5}$. This binding initiates a cascade of events that culminates in differentiation into Th1 cells, by increasing the transcription factor T-bet ${ }^{12}$. Th2 cells have a differentiation process which is dependent on IL-4, which causes the activation of the transcription factor STAT6. This signal together with the T Cell Receptor (TCR) induces the expression of GATA-3. GATA-3 acts as a master regulator of Th2 differentiation ${ }^{12-13}$. GATA-3 and IFN- $\gamma$ R1 subunit expressions were evaluated during different stages of induced periodontal disease (early and advanced) and these data were correlated with the severity of periodontal destruction, which was determined clinically and histologically.

\section{Material and methods}

\section{Study design}

Thirty male adult Wistar rats (Rattus norvegicus albinus), weighing between 300 and $400 \mathrm{~g}$ at the beginning of the experiment and aged approximately 10 weeks, were included in this study. They were kept in polypropylene cages with 5 animals per cage during the whole experimental period and received standard laboratory chow and water ad libitum. The protocol was approved by Institutional Animal Care and Use Committee of the Potiguar University, Brazil (Protocol number: 079/07).

After an adaptation period of 7 days for the animals, experimental periodontitis was induced in animals. Previously, general anesthesia was obtained by intramuscular administration of xylazine hydrochlorate (Virbaxy $1{ }^{\circledR} 2 \%-$ Virbac do Brasil Ind e Com. LTDA - $0.3 \mathrm{mg} / \mathrm{kg}$ ) and ketamine (Francotar ${ }^{\circledR}$ - Virbac do Brasil Ind e Com. LTDA - 1ml/kg). A cotton ligature $\left(\mathrm{n}^{\circ} 10\right.$; Coats Corrente, SP, Brazil) was randomly assigned to one mandibular first molar, in a submarginal position, as previously described ${ }^{14}$. The control side remained unligated and was used as control. Subsequently, the animals were randomly assigned into two groups of 15 animals each:

- Group 1 (initial stage) - ligatures were maintained for 2 days to enable the development of established gingival lesions (G1T - Test Group 1). This period should not induce bone loss on any surface around the tooth. Contralateral teeth were used as controls (G1C - Control Group 1).

- Group 2 (advanced stage) - ligatures were maintained for 15 days (G2T - Test Group 2). This period can cause bone loss (advanced lesions) on any surface around the tooth ${ }^{14}$. Contralateral teeth were also used as controls $(\mathrm{G} 2 \mathrm{C}$ Control Group 2).

Following these protocols, the animals were sacrificed by deep anesthesia and the jaws were removed, dissected and fixed in $10 \%$ neutral formalin for $48 \mathrm{~h}$. Blocks containing the tooth, the inter-radicular bone and soft tissue were obtained. The soft tissues that represent the region of the periodontal pocket were carefully removed with a $15 \mathrm{C}$ scalpel blade. The fragments were processed routinely and stained with hematoxylin and eosin (HE) and for immunohistochemistry. The remaining block containing hard tissue after soft tissue removal was demineralized in a $10 \%$ formic acid solution. This solution was renewed every two days for 12 days, to confirm the presence and absence of bone loss.

\section{Morphological Analysis}

The fragments from soft tissue were embedded in paraffin and histological serial sections of $5 \mathrm{~mm}$ were obtained, followed by $\mathrm{HE}$ staining. Likewise, after the demineralization process, serial sections of $5 \mathrm{~mm}$ from hard tissue were also obtained from the buccolingual position (starting at the buccal bone plate) and stained with HE. After the staining process, the intensity of the inflammatory infiltrate (mild, moderate and intense), the prevailing cell types (neutrophils, lymphocytes, plasma cells, macrophages) and vascularization (scarce, moderate and intense) were analyzed in the soft tissue under an optical microscope (magnification of 400x). Presence or absence of bone loss was also analyzed in hard tissue, by visual examination under an optical microscope.

\section{Immunohistochemical methods}

For the immunohistochemical study, 3- $\mu$ m-thick sections were obtained from paraffin-embedded gingival tissue blocks. The tissue sections were de-paraffinized and immersed in $3 \%$ hydrogen peroxide to block endogenous peroxidase activity. Antigen retrieval was performed in a Pascal pressure cooker with citrate buffer, $\mathrm{pH}$ 6.0, and TRIS/EDTA, for g R1 and GATA-3, respectively. After treatment with normal serum, the sections were incubated with the primary anti-IFN-g R1 antibody diluted 1:100 (Santa Cruz Biotechnology, Santa Cruz, CA, USA) for $18 \mathrm{~h}$ and anti-GATA-3 diluted 1:250 (Santa Cruz Biotechnology, Santa Cruz, CA, USA) for 60 min. The tissue sections were then washed twice in TRIS $\mathrm{pH}$ 7.4 and treated with an immunoperoxidase-based kit (Envision + Dual Link System peroxidase; Dako, Carpinteria, CA, USA) at room temperature to bind the primary antibody. Peroxidase activity was visualized by immersing tissue sections in diaminobenzidine (Liquid DAB + Substrate; Dako, Capinteria, CA, USA) for $3 \mathrm{~min}$, resulting in a brown reaction product. Finally, the tissue sections were counterstained with Mayer haematoxylin and cover slipped. As a negative control, sections of gingival tissues from animals (test and control sides) were treated as earlier, except for the primary antibody, which was replaced by a solution of bovine serum albumin in PBS (Phosphate-Buffered Saline). 


\section{Immunostaining and statistical analysis}

For quantitative analysis, immunostained cells were evaluated for each histological tissue in five fields on each slide; the 5 histological fields were photographed using an Olympus CX41 camera. Images were downloaded and the immunostained cells were counted using an Image analysis system (Image Tool for Windows, version 3.0). The total number of immunostained cells in each field was recorded and the averages corresponding to each slide calculated. Averages were registered for each animal and each group. Data interpretation was performed by a trained examiner, without knowledge of the different groups (blinded study).

A statistical software was used for statistical analysis (SPSS - Statistical Package for Social Sciences, v. 13). Immunohistochemical expression of IFN- $\gamma$ R1 and GATA-3 was compared in the two independent groups, group 1 (2 days) and group 2 (15 days). The Shapiro-Wilk test was performed to confirm the normal distribution of the groups. Levene's F test was applied for verification of equal variances. To compare inter and intra-group differences, Student's t test was used and the Mann-Whitney and Wilcoxon tests were used for groups without normal distribution. The level of significance was set at $5 \%$.

\section{Results}

\section{Morphological Data}

Most of the samples from the G1C (Control Group 1) and G2C (Control Group 2) groups exhibited a mild inflammatory infiltrate that was compatible with clinically healthy gingiva. Lymphocytes were identified as the predominant cells and bone loss was not observed in the furcation or interproximal region. For the G1T (Test Group 1), 7 cases exhibited moderate inflammatory infiltration and 8 cases demonstrated intense infiltration. In this group, the infiltrate was predominantly lymphocytic, and foci of osteoclastic cells and bone resorption were observed in the furcation and interproximal regions in 4 cases. The G2T (Test Group 2) presented mild (3 cases), moderate (7 cases) and intense infiltration (5 cases). In this group, lymphocytes predominated in seven cases and lymphoplasmacytic infiltrate predominated in 6 cases, bone resorption was observed in all cases. Neutrophils were observed in $100 \%$ cases in the test groups.

\section{Immunohistochemical Data}

All samples showed staining for each antibody. IFN-g R1 was detected in the cytoplasm of all inflammatory cells (Figure 1A) and GATA-3 positivity was found in the lymphocytes (Figure 1B). For the expression of GATA-3, intragroup analysis (G1C/G1T, G2C/G2T) was statistically significant $(\mathrm{p}<0.05)$, indicating a higher expression of GATA-3 in ligated teeth (test side). Positive cells for GATA3 expression were found in groups 1 (means: control side = 45.83/test side $=149.27$ ) and 2 (means: control side $=$ 30.37/test side $=89.84$ ).

These results were compatible with the greater

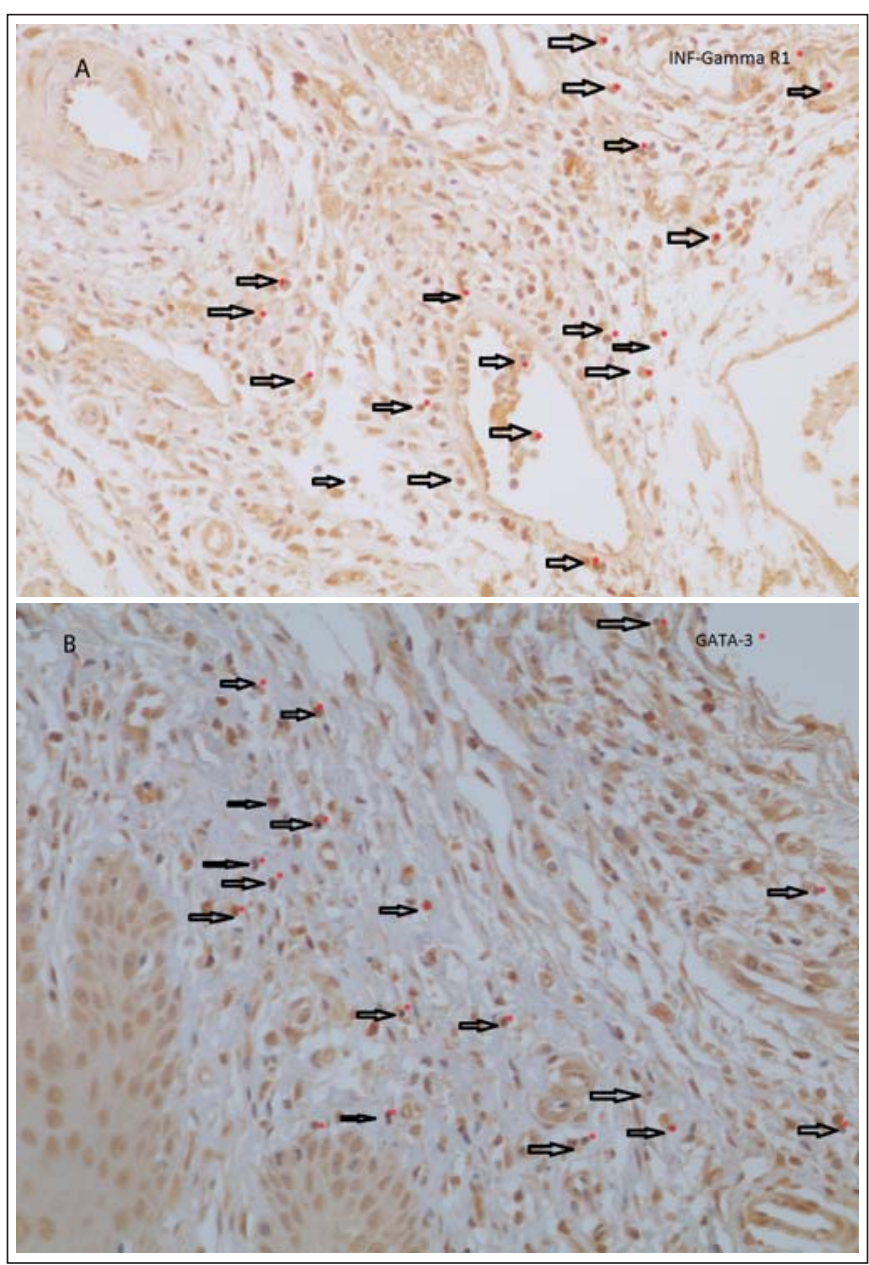

Fig. 1. Test Group: $1 A$ - inflammatory infiltrate showing immunostaining to IFN- $\gamma$ R1 subunit of the receptor for IFN- $\gamma(200 \mathrm{x})$. 1B - Iymphocytic infiltrate showing intracytoplasmic immunostaining to the transcription factor GATA-3(400x).

inflammatory response seen in these groups. With regards to GATA-3 expression, no statistically significant differences were observed comparing the control groups (G1C/G2C). Between the test groups (G1T/G2T), a higher expression was observed in G1T $(p<0.05)$. These results indicate that duration of the cotton ligature could have a significant effect on the expression of GATA-3.

Intragroup analysis $(\mathrm{G} 1 \mathrm{C} / \mathrm{G} 1 \mathrm{~T}, \mathrm{G} 2 \mathrm{C} / \mathrm{G} 2 \mathrm{~T})$ for IFN- $\gamma \mathrm{R} 1$ showed statistically significant differences between the test and control sides $(\mathrm{p}<0.05)$, demonstrating an increased expression of this receptor in ligated teeth (test side). Positive cells for IFN- $\gamma$ R1 expression in groups 1 (means: control side $=66.25 /$ test side $=138.98$ ) and 2 (means: control side $=$ $61.44 /$ test side $=102.11$ ). However, no statistical differences were observed between test groups (G1T/G2T), i.e., the ligation time was not decisive for IFN- $\gamma$ R1 expression. In addition, differences were not observed between the control groups (G1C/G2C).

\section{Discussion}

The interaction of bacterial challenge and host response induces the expression of several molecules, including 
metalloproteinases, involved in the process of destructive periodontal disease ${ }^{15}$ and cytokines ${ }^{5,16-17}$. The cytokines exhibit a strong regulatory role in immune response, regulating the differentiation of native $\mathrm{T}$ cells into Th1 and Th2 cells. Numerous cytokines are produced during the immune response, where IL-12 and IFN- $\gamma$ are involved in the development of the Th1 and Th2 cytokines, and IL-4 induces the differentiation of Th2 cells ${ }^{17}$. Other cytokines are also directly involved in the pathogenesis of periodontal disease, such as interleukin 6 (IL-6), interleukin 8 (IL-8) and tumor necrosis factor $\alpha(\mathrm{TNF}-\alpha)^{16}$.

The pathogenesis of periodontitis is classically described as stable and destructive. Based on the paradigm of Th1/Th2 cells, periodontal lesions with a predominantly Th1 profile may be termed as a stable lesion (gingivitis). In the lesions with a predominance of Th2 cells, a destructive profile (periodontitis) may be present ${ }^{18}$. However, some authors have shown a protective role of the Th2 cells ${ }^{10,19-20}$. Since the exact knowledge of this response remains unclear, the object of this study was to analyze by immunohistochemistry the expression of Th2 cells in the destructive phase of periodontal disease as well as to evaluate possible differences in the expression of surface IFN- $\gamma$ R1. The expression of this receptor is crucial for an effective Th1 response during the evolution of this model of experimentally induced periodontal disease in rats, which has been widely used in several studies ${ }^{21-26}$.

In the control groups, presence of a mild lymphocytic cellular infiltrate was typically observed. In absence of ligatures these cells are a possible indicator of a constant response to bacterial challenge in the gingival sulcus, as described in other studies $^{23}$. In the experimental groups, an acute cellular inflammatory response was observed; during the first 2 days of ligature, the cellular infiltrate became moderate to intense and remained even after 15 days. Similar results were observed in human specimens ${ }^{27}$, where the authors analyzed the immunohistochemical phenotype of cells participating in the immune response in gingivitis and periodontitis. No statistically significant differences were observed in relation to $\mathrm{T}$ and $\mathrm{B}$ lymphocyte densities or in the number of dendritic cells in the described inflammatory conditions.

Regarding the type of cellular infiltrate in the test groups, lymphocytes and plasma cells predominated, whereas in controls groups the lymphocytes were the predominant cells. However, the presence of neutrophils was frequent in all test groups (G1T, G2T). This indicates that the ligatures were able to induce an inflammatory response and that the total number of cells remained during disease progression. As described in the literature, cell density ( $\mathrm{T}$ and $\mathrm{B}$ cells) can remain unchanged between different inflammatory conditions, such as gingivitis and periodontitis ${ }^{27}$. Other studies attributed a pathological role for B cells in periodontal disease $^{5,28}$. These findings imply a greater involvement of Th2 cells at this stage of disease, since Th2 cytokines such as IL-4, IL-5, IL-6 stimulate the proliferation and differentiation of B cells ${ }^{29}$.

The results of this study demonstrate the involvement of Th2 cells in the test groups, i.e., at the stage of the increased inflammatory response in experimental periodontitis. Bone loss was predominantly observed at 15 days in the G2T group as well as initial foci of bone resorption in 4 cases of the G1T group. This finding indicates that in this experimental model of disease the inflammatory process installs quickly, with consequent destruction of periodontal tissue. It probably makes this model ideal for the study of acute and destructive phases of periodontal disease. Some authors ${ }^{23,30}$ reported that the presence of the ligature around teeth induces an acute inflammatory response, resulting in bone loss and loss of connective tissue attachment.

The active phase of experimental periodontal disease was consistent with the increased expression of GATA-3, compared to the control groups, suggesting a greater involvement of Th2 cells in the higher inflammatory response phase. Several studies have demonstrated that the periodontitis lesion involves predominantly B cells; this implies a greater involvement of Th2 cells in the destructive phase of the disease ${ }^{18,31}$. However, when experimental groups were compared, i.e. when the course of the disease was evaluated, the expression of GATA-3 was found to decrease significantly $(p<0.05)$, together with a reduction in the amount of Th2 cells $(\mathrm{G} 1 \mathrm{~T}=149.27 / \mathrm{G} 2 \mathrm{~T}=89.84)$. With this reduction, the anti-inflammatory Th2 responses are probably smaller. Some authors have shown a protective role of the Th2 cells ${ }^{10,19-20,32-34}$. There is an association between the absence of IL-10 (lower Th2 response) with the presence of severe bone loss induced by $P$. gingivalis ${ }^{19}$ and patients with chronic periodontitis have suppressed anti-inflammatory cytokine production that can be partly restored by neutralizing pro-inflammatory cytokines, showing a cross-talk between the production of these cytokines ${ }^{33}$. This protective role was described in a clinical study that measured the levels of several cytokines, including IL-4, before and after nonsurgical periodontal therapy in humans. The levels of IL-4 significantly increased following treatment, contributing to reduce periodontal inflammation ${ }^{32}$. However, a destructive role is also assigned to these cells, since they can reduce the Th1 response, resulting in failure to control the infection ${ }^{35}$. This contradictory role of Th cells can be explained by the conversion of Th1 to Th2 cells, influenced by epigenetic modifications in the environment ${ }^{36}$.

It is clear that a group of $\mathrm{T}$ cells with destructive role, named Th17, play an important role in osteoclast formation. The involvement of these cells in periodontal disease has been reported ${ }^{5,34}$. The results show that in periodontal lesions, IL-17 levels are significantly greater than IL-4, which plays a protective role in the pathogenesis of periodontal disease ${ }^{34}$.

Based on the results of the present study and the reviewed literature, it may be suggested that the decreased expression of GATA-3 (lower number of Th2 cells) led to a reduction in the anti-inflammatory potential in the microenvironment, which culminated with the greatest bone loss seen in the G2T group. As this model seems to stabilize over time ${ }^{23}$, it may be suggested that studies involving a longer period of observation may result in the return of this marker expression.

Evaluation of the expression of the IFN- $\gamma$ R1 subunit of 
the IFN- $\gamma$ receptor was carried out to observe whether the microenvironment is prepared for the Th1 response. The presence of functional receptors for IFN- $\gamma$ on cells could be associated with the presence of the cytokine in the extracellular environment ${ }^{11}$. Comparisons between the test and control teeth for the IFN- $\gamma$ R1 subunit have shown an increased expression of this subunit during the active phase of periodontal disease. However, inter-group analysis demonstrated that the expression of this receptor did not change. These findings show that there was a direct relationship between increased inflammatory response and IFN- $\gamma$ R1 expression. This result partially agrees with another author ${ }^{35}$, who showed that cells carrying IFN- $\gamma$ increased significantly with the expansion of inflammation due to the increase in inflammatory response observed during periodontal tissue destruction. However, in the present study, the expression of the IFN- $\gamma$ R 1 subunit did not change with increased bone loss. These results demonstrate that with the progression of periodontal disease the expression of this receptor remained unchanged. This can also be explained based on the cell types that express this receptor.

The IFN- $\gamma \mathrm{R} 1$ subunit of the IFN- $\gamma$ receptor is not exclusive for Th cells $^{37}$ and it is moderately expressed in almost all inflammatory cells or non-inflammatory cells, where gene expression of the R1 chain appears to be constitutive in these cell types. The analysis of the promoter region of this gene revealed a structure that resembles that of the "housekeeping" genes (genes involved in basic functions of the cells and that are constitutively expressed). These authors also stated further that the expression of this subunit of the IFN- receptor is not regulated by external stimuli, unlike the R2 subunit, which can be regulated positively or negatively depending on the stimulus. Analyzing this information, the R1 subunit of the receptor for IFN- $\gamma$ did not work as an indicator of the presence of Th1 cytokines in the microenvironment. When these results are compared with those of the literature ${ }^{37-38}$, it is accepted that this subunit is not regulated by the presence of the cytokine in the environment, since several other inflammatory cell types constitutively express this subunit.

Therefore, it may be suggested that the development of the inflammatory response was associated with the increase in IFN- $\gamma$ expression, due to the increased number of inflammatory cells that carry this subunit. In addition, the expression of this subunit did not change between the test groups, as the amount of the inflammatory infiltrate also remained the same. Regulatory mechanisms of the R1 subunit have been described ${ }^{37}$ after antigen recognition that there is a temporary down-regulation in native $\mathrm{T}$ cells. Without this subunit, the cross-talk with the cytokine does not occur, and the antiproliferative effects associated with IFN- $\gamma$ are nullified. This may explain the slight reduction in the expression of this subunit in the G2T in the present study. As described, the participation of Th2 cells in periodontitis was observed herein; however, it is still unclear whether these cells are more protective or destructive in the context of periodontal pathogenesis, since they exhibit conflicting roles, i.e., they may be either protective or destructive according to their expression profile.

Some studies have reported the participation of both Th 1 and Th2 cytokines in periodontal disease $\mathrm{e}^{5,39}$. As previously described, these two groups of cytokines play an important role in maintaining the homeostasis of the alveolar bone ${ }^{39}$; however, differences in the balance of cytokines in the environment may result in the progression of periodontal disease ${ }^{7}$.

According to the results of the present study, it may be suggested that the decrease in positive Th2 cells during the phase of higher tissue destruction, associated with experimentally induced periodontitis, may have contributed to the evolution of the destructive process. Additionally, the significant expression of IFN $\gamma \mathrm{R} 1$ subunit in the test group and the lack of alteration in expression observed in the ligated teeth throughout the experiment (between G1T and G2T) suggests that this receptor is expressed at the beginning of inflammatory response development. This alteration makes these microenvironments more prepared for the Th1 response, but there was no association between the presence of this receptor and increased bone loss.

Further experiments are necessary in order to better understand the host response against the biofilm in the periodontal tissues. Understanding the regulatory mechanisms of IFN- $\gamma$ and GATA-3 during the development of T cell lines may also provide a better understanding of the Th1/Th2 response in periodontal disease as well as allow the development of methods of treatment modulation that may be able to balance this response.

In conclusion, the current study demonstrates, in different periods of the active phase of experimental periodontal disease, that the decrease in the expression of the GATA-3 transcription factor was associated with increased bone loss, suggesting that a controlled Th2 response may be associated with protective mechanisms during the active phase of periodontal destruction induced by ligatures in rats and the IFN- $\gamma$ R1 subunit may not be associated with the progression of experimental periodontal disease.

\section{Acknowledgements}

This study was supported by the Post-graduate Program in Oral Pathology of the Federal University of Rio Grande do Norte and part of this research was conducted at the University Potiguar - Department of Dentistry - RN, Brazil.

\section{References}

1. Ianni M, Bruzzesi G, Pugliese D, Porcellini E, Carbone I, Schiavone A, et al. Variations in inflammatory genes are associated with periodontitis. Immun Ageing. 2013; 10:1-8.

2. Yucel-Lindberg T, Båge T. Inflammatory mediators in the pathogenesis of periodontitis. Expert Rev Mol Med. 2013; 15: e7.

3. Kayal RA. The role of osteoimmunology in periodontal disease. Biomed Res Int. 2013; 2013: 1-12.

4. Yamamoto M, Fujihshi K, Hiroi T, McGhee JR, Van Dyke TE, Kiyono H. 
Molecular and cellular mechanisms for periodontal diseases: role of Th1 and Th2 type cytokines induction of mucosal inflammation. J Periodontal Res. 1997; 32: 115-9.

5. Di Benedetto A, Gigante I, Colucci S, Grano M. Periodontal disease: linking the primary inflammation to bone loss. Clin Dev Immunol. 2013; 2013: 1-7.

6. Teng YTA. The role of acquired immunity and periodontal disease progression. Crit Rev Oral Biol Med. 2003; 14: 237-52.

7. Okoye IS, Wilson MS. CD4+ T helper 2 cells - microbial triggers, differentiation requirements and effector functions. Immunology. 2011; 134 : 368-77.

8. Glowacki AJ, Yoshizawa S, Jhunjhunwala S, Vieira AE, Garlet GP, Sfeir $C$, et al. Prevention of inflammation-mediated bone loss in murine and canine periodontal disease via recruitment of regulatory lymphocytes. Proc Natl Acad Sci USA. 2013; 12: 18525-30.

9. Garlet GP. Destructive and protective roles of cytokines in periodontitis: A re-appraisal from host defense and tissue destruction viewpoints. J Dent Res. 2010; 89: 1349-63.

10. Fu QY, Zhang L, Duan L, Qian SY, Pang HX. Correlation of chronic periodontitis in tropical area and IFN-ã, IL-10, IL-17 levels. Asian Pac J Trop Med. 2013; 6: 489-92.

11. Baker PJ, Boutaugh NR, Tiffany M, Roopenian DC. B Cell IgD Deletion Prevents Alveolar Bone Loss following murine oral infection. Interdiscip Perspect Infect Dis. 2009; 2009: 1-6.

12. Chakir $\mathrm{H}$, Wang $\mathrm{H}$, Lefebvre DE, Webb J, Scott FW. T-bet/GATA-3 ratio as a measure of the Th1/Th2 cytokine profile in mixed cell populations: predominant role of GATA-3. J Immunol Methods. 2003; 278: 157-69.

13. Teng YTA. Mixed Periodontal Th1-Th2 cytokine profile in Actinobacillus actinomycetencomitans - specific osteoprotegerin ligand (or RANK-L) mediated alveolar bone destruction in vivo. Infect Immun. 2002; 70 : 5269-73.

14. Kuhr A, Popa Wagner A, Schmoll H, Schwahn C, Kocher T. Observations on experimental marginal periodontitis in rats. J Periodontal Res. 2004; 39: 101-6

15. Nagasupriya A, Rao DB, Ravikanth M, Kumar NG, Ramachandran $\mathrm{CR}$, Saraswathi TR. Immunohistochemical expression of matrix metalloproteinase 13 in chronic periodontitis. Int J Periodontics Restorative Dent. 2014; 34: 79-84.

16. Noh MK, Jung M, Kim SH, Lee SR, Park KH, Kim DH, et al. Assessment of IL-6, IL-8 and TNF-á levels in the gingival tissue of patients with periodontitis. Exp Ther Med. 2013; 6: 847-51.

17. O'Garra A. Cytokines induce the development of functionally heterogeneous T helper cell subsets. Immunity. 1998; 8: 275-83.

18. Gemmell E, Seymour GJ. Immunoregulatory control of Th1/Th2 cytokine profiles in peridontal disease. Periodontology 2000. 2004; 35: 21-41.

19. Sasaki H, Okamatsu Y, Kawai T, Taubman M, Stashenko P. The interleukin10 knockout mouse is highly susceptible to Porphyromonas gingivalisinduced alveolar bone loss. J Periodontal Res. 2004; 39: 432-41.

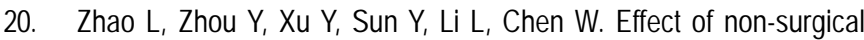
periodontal therapy on the levels of Th17/Th1/Th2 cytokines and their transcription factors in Chinese chronic periodontitis patients. J Clin Periodontol. 2011; 38: 509-16.

21. Graves DT, Fine D, Teng YT, Van Dyke TE, Hajishengallis G. The use of rodent models to investigate host-bacteria interactions related to periodontal diseases. J Clin Periodontol. 2008; 35: 89-105.

22. Cavagni J, Soletti AC, Gaio EJ, Rösing CK. The effect of dexamethasone in the pathogenesis of ligature-induced periodontal disease in Wistar rats. Braz Oral Res. 2005; 19: 290-4.

23. Gurgel BC, Duarte PM, Nociti FH JR, Sallum EA, Casat MZ, Sallum AW, et al. Impact of an anti-inflammatory therapy and its withdrawal on the progression of experimental periodontitis in rats. J Periodontol. 2004; 75: $1613-8$.

24. Galvão MP, Chapper A, Rösing CK, Ferreira MB, De Souza MA.
Methodological considerations on descriptive studies of induced periodontal disease in rats. Pesq Odontol Bras. 2003; 17: 56-62.

25. Souza DM; Ricardo LH; Rocha RF. Effects of alcohol intake in periodontitis progression in female rats: a histometric study. Braz J Oral Sci. 2013; 13 229-34.

26. Oz HS, Puleo DA. Animal models for periodontal diseases. J Biomed Biotechnol. 2011; 1- 8.

27. Lins RD, Figueiredo CR, Queiroz LM, Da Silveira EJ, Freitas RA. Immunohistoquemical evaluation of the inflammatory response in periodontal disease. Braz Dent J. 2008; 19: 9-14.

28. Oliver-Bell J, Butcher JP, Malcolm J, MacLeod MK, Adrados Planell A, Campbell $L$, et al. Periodontitis in the absence of $B$ cells and specific antibacterial antibody. Mol Oral Microbiol. 2015; 30: 160-9.

29. Yamazaki K, Nakajima T, Hara K. Immunohistological analysis of T cell functional subsets in chronic inflammatory periodontal disease. Clin Exp Immunol. 1995; 99: 384-91.

30. Nyman S, Schroeder HE, Lindhe J. Supression of inflammation and bone resorption by indomethacin durin experimental periodontitis in dogs. J Periodontol. 1979; 50: 450-61.

31. Arun KV, Talwar A, Kumar TS. T-helper cells in the etiopathogenesis of periodontal disease: A mini review. J Indian Soc Periodontol. 2011; 15: 4-10.

32. Pradeep AR, Roopa Y, Swati PP. Interleukin-4, a T-helper 2 cell cytokine, is associated with the remission of periodontal disease. J Periodontal Res. 2008; 43: 712-6.

33. Berker E, Kantarci A, Hasturk H, Van Dyke TE. Blocking proinflammatory cytokine release modulates peripheral blood mononuclear cell response to Porphyromonas gingivalis. J Periodontol. 2013; 84: 1337-45.

34. Behfarnia P, Birang R, Pishva SS, Hakemi MG, Khorasani MM. Expression levels of th-2 and th-17 characteristic genes in healthy tissue versus periodontitis. J Dent (Tehran). 2013; 10: 23-31.

35. Ukai T, Mori Y, Onoyama M, Hara Y. Immunohistological study of interferon-gamma- and interleukin-4-bearing cells in human periodontitis gingiva. Arch Oral Biol. 2001; 46: 901-8.

36. Talwar A, Arun KV, Kumar TSS, Clements J. Plasticity of T helper cell subsets: Implications in periodontal disease. J Indian Soc Periodontol. 2013; 17: 288-91.

37. Bach EA, Aguet M, Schreiber RD. The IFN gamma receptor: a paradigm for cytokine receptor signaling. Ann Rev Immunol. 1997; 15: 563-91.

38. Skarenta $\mathrm{H}$, Yang Y, Pestka S, Fathman CG. Ligand-independent downregulation of IFN-? Receptor 1 following TCR engagement. J Immunol. 2000; 164: 3506-11.

39. Alayan J, Ivanovski S, Farah CS. Alveolar bone loss in T helper $1 / \mathrm{T}$ helper 2 cytokine-deficient mice. J Periodontal Res. 2007; 42: 97-103. 